\title{
Effect of Vibrations on Pore Fluid Distribution in Porous Media
}

\author{
MING XIAO ${ }^{1, \star}$, LAKSHMI N. REDDI $^{1}$ and SUSAN L. STEINBERG ${ }^{2}$ \\ ${ }^{1}$ Civil Engineering Department, Kansas State University, Manhattan, KS 66506, U.S.A. \\ ${ }^{2}$ Universities Space Research Association, NASA Johnson Space Center, Houston, TX \\ 77058, U.S.A.
}

(Received: 27 February 2004; in final form: 28 January 2005)

\begin{abstract}
Understanding the role of shuttle vibrations in pore fluid distribution is an essential task in the exploration of plant growth in root modules aboard space flights. Results from experimental investigations are reported in this paper on the distribution of immiscible fluid phases in glass beads under vibrations. Hexadecane, a petroleum compound immiscible with and lighter than water, was used in the experiments. The higher freezing point of Hexadecane $\left(18^{\circ} \mathrm{C}\right)$ allowed the solidification of the entrapped blobs in the presence of water in porous media, so that their size distribution can be obtained. van Genuchten function, commonly used to express moisture retention curves, is found to be an adequate fit for blob size distribution at residual saturation. The effect of vibrations on the fate (mobilization, stranding, or breakup) of a solitary ganglion in porous media was studied using a network model. A mobility criterion considering viscous, gravity, and capillary forces was developed to determine the fate of a solitary ganglion in a porous medium. It is concluded that the effect of vibrations is to increase the likelihood of breakup and mobilization of blobs entrapped in porous media at residual saturation. The pore fluid distributions after vibrations are less uniform than those before vibrations.
\end{abstract}

Key words: vibrations, pore fluid distribution, network model.

\section{Introduction}

Distribution of water and air in soils is a common problem of interest in several contexts of soil sciences and geotechnical engineering. Plant growth in soils requires good understanding of the availability of wetting fluid (water) to root systems. The present study is part of a NASA project aimed at developing an improved understanding of the conditions required for plant growth in zero and micro-gravity conditions. In addition to the changes in gravitational force, plant growth modules in space are influenced by vibrations during launch and orbital movement in space. The

\footnotetext{
`Author for correspondence: mingxiao@ksu.edu
} 
purpose of this paper is to report results from experimental and modeling investigations on how vibrations affect discontinuous fluid phase mobilization and distribution in porous media.

Distribution of discontinuous pore water in soils has seldom been reported in the literature, perhaps due to the difficulties in measuring the characteristics of water blobs in pores. However, distribution of oil ganglia in porous media has been widely investigated in oil recovery processes in petroleum engineering and in groundwater remediation technologies in geoenvironmental engineering. The blob size distribution (BSD) of nonaqueous phase liquid (NAPL) can be used to study the distribution of immiscible phases, such as water and air, in soils. For example, Mayer and Miller (1992) derived the BSD of styrene monomer, a type of NAPL, in glass beads. They polymerized the styrene monomer in glass beads, and the solid styrene monomer blobs were sieved to obtain the BSD. Mayer and Miller (1992) also proposed to fit the NAPL blob size distribution with the Van Genuchten function

$$
F(d)=1-\left[1+(\beta d)^{m}\right]^{(1 / m)-1},
$$

where $d$ is the blob size $(\mathrm{mm}), F(d)$ the mass percentage of blobs that are finer that size $d, \beta$ and $m$ are fitting parameters, $\beta\left(\mathrm{mm}^{-1}\right)$ increases with the decrease of mean blob sizes, $m$ is related to the shape of the BSD curve ( $m$ is larger for more uniformly distributed blob sizes).

In order to study the distribution and transport of immiscible ganglia in granular porous media, it is essential to understand the mechanisms involved in the movement of a solitary ganglion. Depending on the external forces it is subjected to, a single ganglion in a porous medium can be mobilized, stranded, or broken up into more than one daughter ganglion. To determine the fate of a solitary ganglion in a porous medium, mobility criteria should be established. Ng and Payatakes (1980) developed a ganglion mobilization criterion in which viscous force is considered as the primary driving force. The criterion was expressed as

$$
\begin{aligned}
\frac{\mu_{\mathrm{w}} v_{\mathrm{f}}}{\gamma_{\mathrm{ow}}}>\frac{k_{\mathrm{rw}} k}{\beta_{K I}} \rightarrow \text { mobilization, } \\
\frac{\mu_{\mathrm{w}} v_{\mathrm{f}}}{\gamma_{\mathrm{ow}}}<\frac{k_{\mathrm{rw}} k}{\beta_{K I}} \rightarrow \text { stranding, }
\end{aligned}
$$

where $\mu_{\mathrm{w}}$ is the absolute viscosity of water, $v_{\mathrm{f}}$ is the flow velocity, $\gamma_{\mathrm{ow}}$ is the interfacial tension between water and the oil ganglion, $k_{\mathrm{rw}}$ is the relative permeability to water when the porous medium is not fully water-saturated due to ganglia occupation in the porous medium, $k$ is the saturated intrinsic permeability, and $\beta_{K I}$ is the maximum appendix mobility factor that is determined by the geometry of pore throats containing the ganglion. 
A simplified description of pore geometry allowed Reddi and Challa (1994) to express ganglion mobility criterion under viscous and gravity forces as

$$
\begin{aligned}
& \frac{\Delta \rho g L_{\mathrm{v}} d_{\mathrm{t}}}{\gamma_{\mathrm{ow}}}>4 \rightarrow \text { mobilization due to gravity, } \\
& \frac{\mu_{\mathrm{w}} V_{\mathrm{f}} L_{\mathrm{f}} d_{\mathrm{t}}}{k \gamma_{\mathrm{ow}}}>4 \rightarrow \text { mobilization due to viscous force, }
\end{aligned}
$$

where $\Delta \rho$ is the density difference between water and the NAPL, $g$ is the acceleration due to gravity, $L_{\mathrm{V}}$ the vertical length of the ganglion, $L_{\mathrm{f}}$ is the length of the ganglion in the direction of flow, $k$ the intrinsic permeability of the porous medium, and $d_{\mathrm{t}}$ is the mean pore throat diameter.

In this paper, an experimental study undertaken to investigate the effect of vibrations on discontinuous pore fluid distribution is reported first. Then the effect of vibrations on the fate (mobilization, stranding, or breakup) of a solitary ganglion in porous media is documented using a network model reported in the literature by applying a mobility criterion developed in this study.

\section{Experimental Investigations}

In this study, the water-air fluid distribution is addressed using methods reported in the NAPL literature. To measure the pore fluid distribution under idealized conditions of pore geometry, glass beads were used as porous media. Five different glass beads samples were used: (1) $0.8 \mathrm{~mm}$ uniform glass beads; (2) $0.8 \mathrm{~mm}$ and $1.5 \mathrm{~mm}$ combined with equal masses; (3) 1.2 and $2.0 \mathrm{~mm}$ combined with equal masses; (4) $2.0,3.0,4.0 \mathrm{~mm}$ combined with equal masses; (5) 4.0, 5.0, 6.0 $\mathrm{mm}$ combined with equal masses. To obtain the measurements on discontinuous fluid phase distributions in glass beads, Hexadecane, a petroleum compound immiscible with and lighter than water, was used in the experiments. The higher freezing point of Hexadecane $\left(18^{\circ} \mathrm{C}\right)$ allowed the solidification of the entrapped blobs in the presence of water in porous media, so that their size distribution can be obtained by wet-sieving the solid blobs. The BSD of Hexadecane is the size distribution of Hexadecane blobs that are entrapped in the glass beads under residual Hexadecane saturation. Using Hexadecane as a surrogate fluid, the effects of vibrations on discontinuous fluid phase distributions were studied. van Genuchten expression proposed by Mayer and Miller (1992) in Equation (1) allowed us to quantify the effects of vibrations. Before the test, the colorless Hexadecane was dyed red using an organic dye (Oil Red O, from Sigma Corp) to distinguish it from water. 
The procedure used to obtain Hexadecane BSD is as follows:

(1) Pump water into dry glass beads at a flow rate of $50 \mathrm{ml} / \mathrm{min}$ until the glass beads are fully saturated and $2 \mathrm{~cm}$ high free water is left above the glass beads.

(2) Slowly pour in Hexadecane on top of the free water. Then apply suction at the bottom of the glass beads using a programmable pump until the glass beads are fully saturated with Hexadecane. A suction flow rate of $15 \mathrm{ml} / \mathrm{min}$ is used.

(3) Flood water back into glass beads. Most Hexadecane is flooded out of the glass beads sample. The Hexadecane entrapped in the pores of glass beads forms the residual saturation. A flooding flow rate of $100 \mathrm{ml} / \mathrm{min}$ is used at this step.

(4) Freeze the Hexadecane at a temperature between $0{ }^{\circ} \mathrm{C}$ and $18{ }^{\circ} \mathrm{C}$ in the presence of water.

(5) Collect the Hexadecane blobs, and wet sieve them using water colder than $18^{\circ} \mathrm{C}$ to obtain blob size distribution.

Figure 1 shows the Hexadecane BSDs in the five glass beads samples. The results illustrate the expected result that porous media with smaller grain sizes are associated with smaller blobs. The Van Genuchten function (Equation (1)) was used to fit these BSDs. Figure 2 shows representative

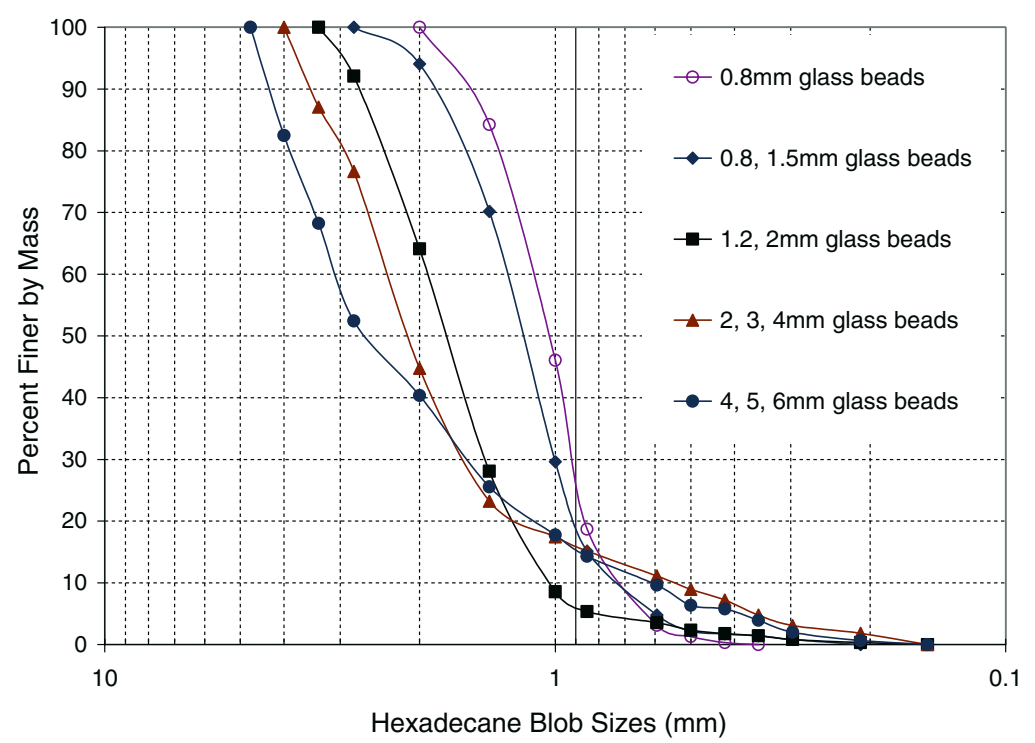

Figure 1. Hexadecane blob size distributions in glass beads (NO vibrations). 


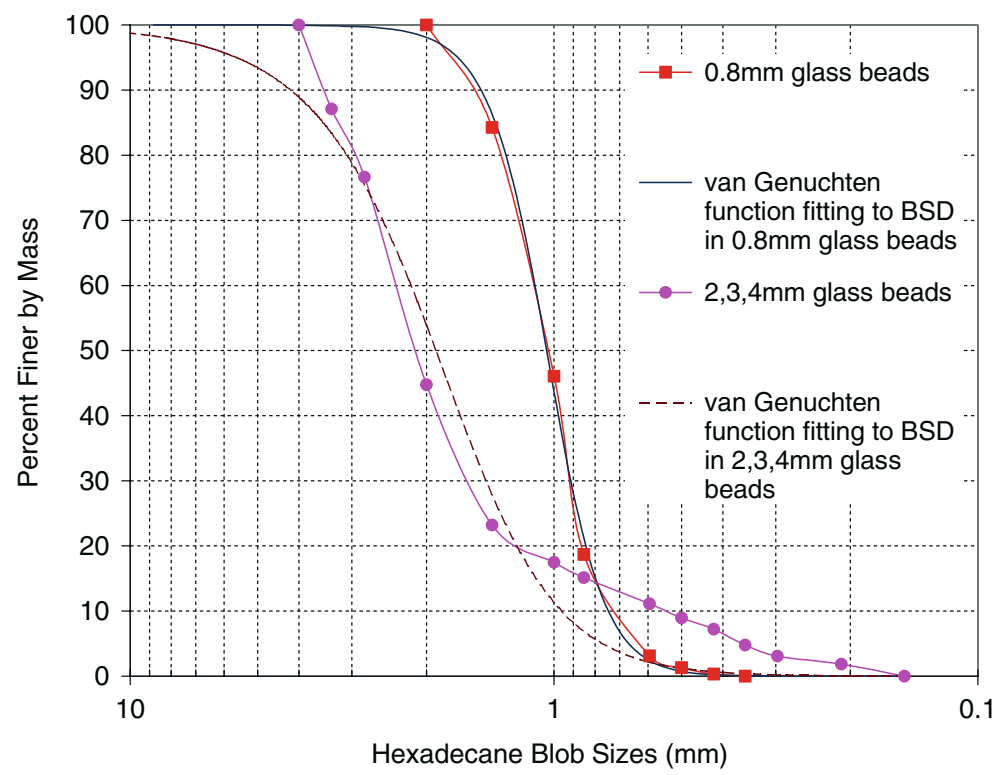

Figure 2. Van Genuchten function fitting to blob size distributions in $0.8 \mathrm{~mm}$ glass beads and in 2, 3, $4 \mathrm{~mm}$ glass beads.

fits to BSDs in $0.8 \mathrm{~mm}$ glass beads sample and in 2, 3, and $4 \mathrm{~mm}$ glass beads sample. The Van Genuchten function yielded a satisfactory fit to the experimental results; the fit is better in the case of small glass beads such as $0.8 \mathrm{~mm}$ than in the case of glass beads larger than $2 \mathrm{~mm}$.

In the next series of tests, the glass beads with discontinuous Hexadecane blobs were vibrated using a shaking table (Model: VP51D1; Frequency $60 \mathrm{~Hz}$ or 3600 vibrations/min; Amplitude: $0.33 \mathrm{~mm}$; Manufacture: FMC Technologies) in order to investigate the effect of vibrations on pore fluid distribution. To facilitate comparison of BSDs with and without vibrations, the vibration experiments followed the same procedure of vibration-free tests up to Step 4. After Step 4, the following additional steps were conducted:

(5) Drain the water out of the glass beads, while the solid Hexadecane remains in the pores. The purpose of draining water is to retain only one fluid (wetting) in the sample at residual saturation.

(6) Keep the Plexiglas column, which contains the glass beads and solid residual Hexadecane blobs, above $18^{\circ} \mathrm{C}$ until the Hexadecane completely melts.

(7) Vibrate the Plexiglas column on the shaking table for $30 \mathrm{~s}$.

(8) Freeze the Hexadecane and collect the blobs. Wet sieve the Hexadecane blobs using water colder than $18^{\circ} \mathrm{C}$ to obtain the blob size distribution. 


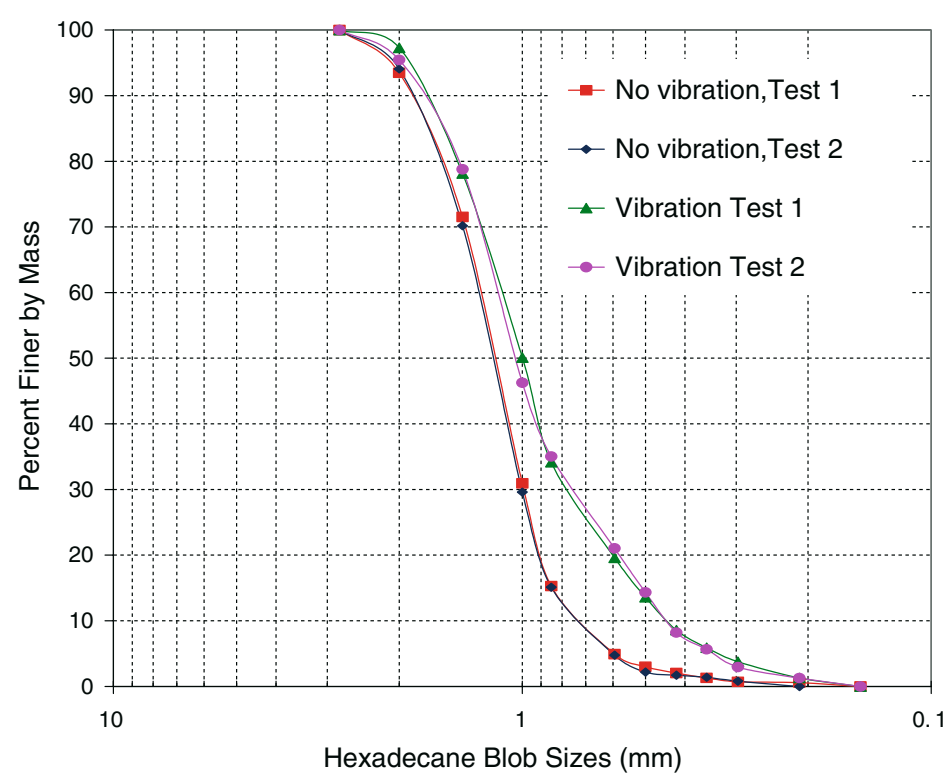

Figure 3. Blob size distributions in glass beads $(0.8,1.5 \mathrm{~mm})$ with and without vibrations.

Figure 3 shows the blob size distributions obtained with and without vibrations in glass beads of sizes 0.8 and $1.5 \mathrm{~mm}$. For each test condition, two identical tests (labeled as Test 1 and Test 2) were conducted to test the reproducibility. The results clearly show the blobs in glass beads after vibrations are smaller than those without vibrations, due to the compacted pores and the resulting breakup of the blobs entrapped in the pores. The results also indicate that the BSDs are highly reproducible in these media. Figure 4 shows the BSDs in glass beads of 1.2 and $2 \mathrm{~mm}$, and it revealed the same vibration effect. The Van Genuchten function fitting is also shown in this figure. Van Genuchten function was used to fit all BSD curves obtained in this study. The resulting parameters are listed in Table I. An increase in $\beta$ indicates the decrease of mean size of entrapped blobs in the pores; a decrease in $m$ indicates that the BSD is less uniformly distributed. Table I shows that the effect of vibrations is to increase $\beta$ and to decrease $m$. These results imply that vibrations break up the entrapped blobs and reduce the mean blob size. Size distribution of blobs after vibrations is less uniform than that before vibrations. Although cyclic force induced by vibrations broke up discontinuous pore blobs, densities of glass beads samples changed little. For example, the density of 0.8 and $1.5 \mathrm{~mm}$ glass beads sample remained at $1.52 \mathrm{~g} / \mathrm{cm}^{3}$ before and after vibrations, and the density of 1.2 and $2.0 \mathrm{~mm}$ glass beads sample decreased slightly from 1.54 to $1.49 \mathrm{~g} / \mathrm{cm}^{3}$. 


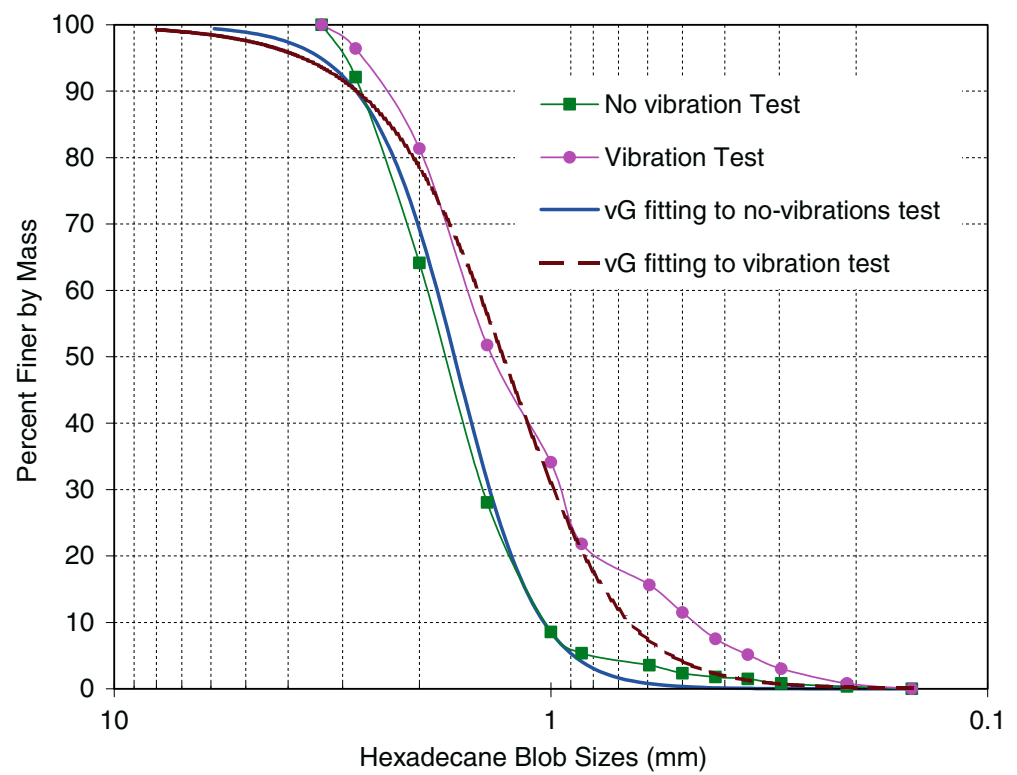

Figure 4. Blob size distributions in glass beads $(1.2$ and $2 \mathrm{~mm})$ with and without vibrations, with the Van Genuchten function fitting.

Table I. Van Genuchten function fitting to blob size distribution (BSD)

\begin{tabular}{|c|c|c|c|c|}
\hline \multirow[t]{2}{*}{ Glass beads $(\mathrm{mm})$} & \multicolumn{2}{|c|}{$\mathrm{BSD}$, no vibrations } & \multicolumn{2}{|c|}{ BSD, with vibrations } \\
\hline & $\beta\left(\mathrm{mm}^{-1}\right)$ & $m$ & $\beta\left(\mathrm{mm}^{-1}\right)$ & $m$ \\
\hline 0.8 & 0.9943 & 6.7482 & N/A & N/A \\
\hline $0.8,1.5$ & 0.9002 & 5.4866 & 1.1764 & 3.6613 \\
\hline $1.2,2$ & 0.6442 & 4.8451 & 0.896 & 3.491 \\
\hline $2,3,4$ & 0.6109 & 3.4247 & 1.0538 & 2.2855 \\
\hline $4,5,6$ & 0.5871 & 2.9172 & 1.5535 & 2.5726 \\
\hline
\end{tabular}

\section{Theoretical Investigations}

The purpose of this theoretical investigation is to provide a better understanding of the mechanisms that are responsible for the mobility (mobilization, breakup, and stranding) of pore fluid blobs. Based on the work of $\mathrm{Ng}$ et al. (1978), $\mathrm{Ng}$ and Payatakes (1980), and Reddi and Challa (1994), we developed below a mobility criterion that takes into account viscous, gravity, and capillary forces to determine the mobility of a solitary pore fluid ganglion. 
(a)
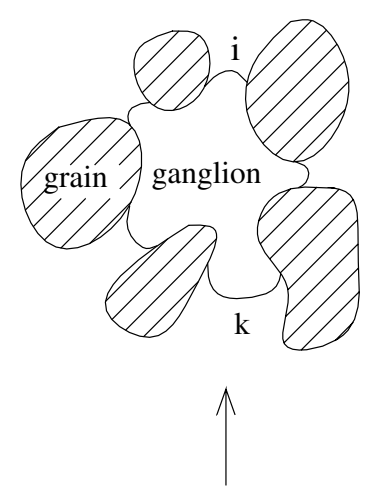

$\triangle \mathrm{p}$ (b)

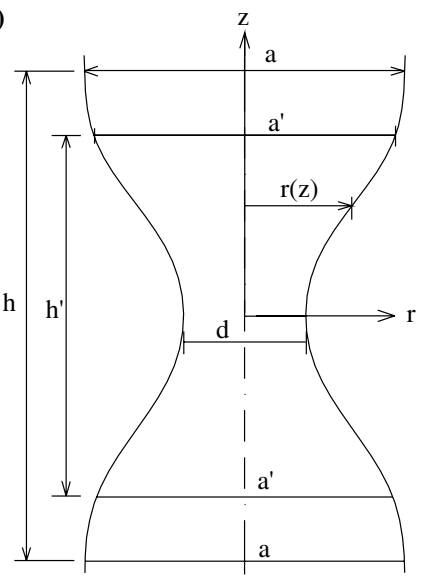

(c)

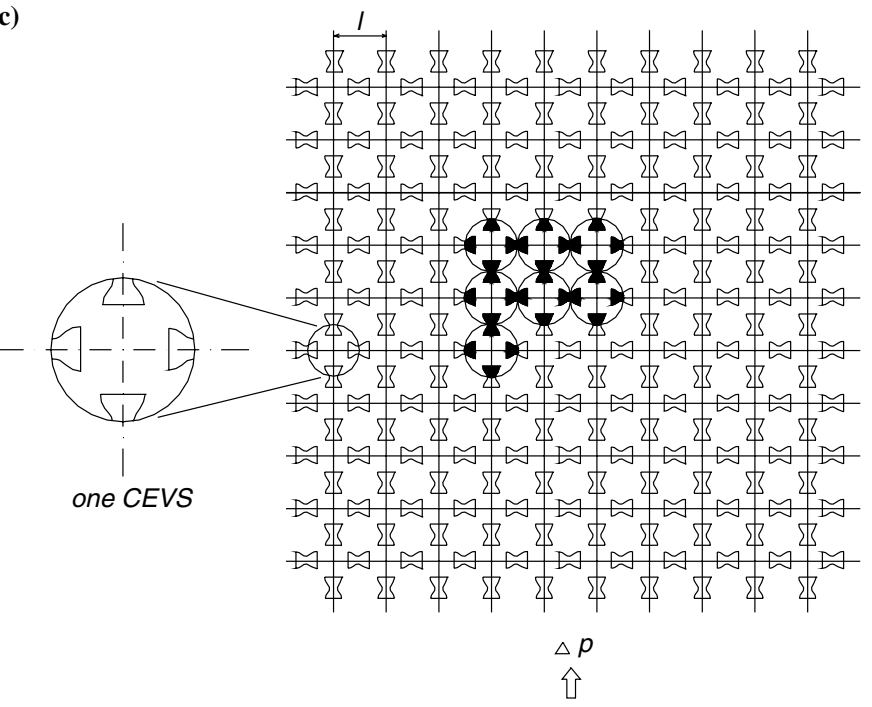

Figure 5. Two-dimensional network model (a) $2 \mathrm{D}$ idealization of a typical ganglion in porous medium. (b) Typical unit cell of the $2 \mathrm{D}$ network model $\left(-h^{\prime} / 2 \leqslant z \leqslant h^{\prime} / 2\right)$. The segment $(-h / 2 \leqslant z \leqslant h / 2)$ is the extended unit cell. (from Payatakes et al., 1980). (c) 2D network configuration (From $\mathrm{Ng}$ et al., 1980).

\subsection{MOBILITY CRITERION OF SOLITARY GANGLION IN POROUS MEDIA}

Figure 5a shows a solitary ganglion in a pore formed by grains and the direction of the pressure gradient, which is imposed by upward flowing wetting fluid (water). If the NAPL is lighter than water, the external pressure on the ganglion between an arbitrary upstream interface (indexed by $k$ ) and an arbitrary downstream interface (indexed by $i$ ) due to viscous and buoyancy forces is 


$$
p_{\mathrm{ext}}=G L_{\mathrm{v}}+\Delta \rho g L_{\mathrm{v}}
$$

where $G$ is the pressure gradient, $\Delta \rho$ is the density difference between water and the NAPL; $L_{\mathrm{v}}$ is the vertical length between the interfaces indicated by $k$ and $i$. Pressure gradient $G$ can be expressed as

$$
G=\rho g \cdot i,
$$

where $i$ is the hydraulic gradient. According to Darcy's Law

$$
v=K i,
$$

where hydraulic conductivity $K$ can be expressed as

$$
K=\frac{k \rho g}{\mu_{\mathrm{w}}}
$$

$k$ is the intrinsic permeability, and $\mu_{\mathrm{w}}$ is the absolute viscosity of water. Equation (6) may now be written as

$$
p_{\text {ext }}=\frac{v \mu_{\mathrm{w}}}{k} L_{\mathrm{v}}+\Delta \rho g L_{\mathrm{v}} .
$$

Due to the flow of a continuous fluid in the neighborhood of a ganglion, an external field of viscous stresses is set up. The ganglion responds to this external field by shifting its position slightly in its respective pore throats. Each throat in a downstream direction becomes more curved by pushing ahead toward the throat constriction, and each upstream pore throat becomes less curved by drawing further away from the throat constriction. When the downstream curve reaches the limit of the throat constriction at which interfacial tension can no longer hold the meniscus stable, the ganglion advances to an adjacent pore. The critical condition for the mobilization of a ganglion is that the external pressure difference between the upstream and downstream ends of the ganglion just exceeds the net capillary pressure difference that the two ends can develop by merely shifting in the pores they occupy. The net capillary pressure difference between the menisci of the two ends is

$$
\Delta p_{\mathrm{c}}=p_{\mathrm{c}}^{i}-p_{\mathrm{c}}^{k}=\frac{4 \gamma \cos \theta}{d_{i}}-\frac{4 \gamma \cos \theta}{d_{k}},
$$

where $\gamma$ is the interfacial tension between water and NAPL, $\theta$ is the contact angle of the wetting fluid (water), $d_{i}$ is the downstream pore throat diameter, and $d_{k}$ is the upstream pore throat diameter. The curvature of a meniscus can be expressed as

$$
J=\frac{2 \cos \theta}{d},
$$


where $d$ is the pore throat diameter. The net capillary pressure difference may be expressed as

$$
\Delta p_{\mathrm{c}}=2 \gamma\left(J_{i}-J_{k}\right),
$$

where $J_{i}$ is the curvature of a downstream pore throat, and $J_{k}$ is the curvature of an upstream pore throat. The mobilization criterion may now be simply expressed as

$$
p_{\text {ext }}>\Delta p_{\mathrm{c}}
$$

Substituting Equations (10) and (13) in (14), we have

$$
\frac{v \mu_{\mathrm{w}}}{k} L_{\mathrm{v}}+\Delta \rho g L_{\mathrm{v}}>2 \gamma\left(J_{i}-J_{k}\right)
$$

which can also be written as

$$
\frac{v \mu_{\mathrm{w}}}{\gamma}+\frac{\Delta \rho g k}{\gamma}>\frac{k}{\frac{L_{\mathrm{v}}}{2\left(J_{i}-J_{k}\right)}},
$$

where $L_{\mathrm{v}} / 2\left(J_{i}-J_{k}\right)$ is defined as appendix mobility factor $\left(\beta_{k i}\right)(\mathrm{Ng}$ and Payatakes, 1980), i.e.,

$$
\beta_{k i}=\frac{L_{\mathrm{v}}}{2\left(J_{i}-J_{k}\right)} .
$$

As long as the external pressure exceeds the net capillary pressure difference between any pair of downstream and upstream ends, the ganglion can be mobilized. Define

$$
\beta_{\max }=\max \left\{\beta_{k i} ; i \in \bar{D}, k \in \bar{U}\right\},
$$

where $\bar{D}$ is the domain of downstream pore throat index, and $\bar{U}$ is the domain of upstream pore throat index. So the ganglion mobility criterion when flow is upward is

$$
\begin{aligned}
& N_{\mathrm{ca}}+N_{\mathrm{b}}>\frac{k}{\beta_{\max }} \quad \rightarrow \text { mobilization, } \\
& N_{\mathrm{ca}}+N_{\mathrm{b}} \leqslant \frac{k}{\beta_{\max }} \quad \rightarrow \text { stranding }
\end{aligned}
$$

where capillary number is $N_{\mathrm{ca}}=\frac{v \mu_{\mathrm{w}}}{\gamma}$

and bond number is $N_{\mathrm{b}}=\frac{\Delta \rho g k}{\gamma}$.

When flow is downward, the ' + ' sign in formulae (19) and (20) can be simply changed to '-'. This study assumes the flow is parallel to the direction of gravity in order to derive a simplified form of the mobility criterion. If 
the flow direction is arbitrary, the projected length of a ganglion on the flow direction does not equal the vertical length, so Equation (15) should be expressed as

$$
\frac{v \mu_{\mathrm{w}}}{k} L_{k i} \cos \theta_{k i}+\Delta \rho g L_{k i} \cos \alpha_{k i}>2 \gamma\left(J_{i}-J_{k}\right),
$$

where $L_{k i}$ is the distance between an upstream throat (indexed by $k$ ) and a downstream throat (indexed by i), $\theta_{k i}$ the angle between vector $\overrightarrow{L_{k i}}$ and the flow direction, and $\alpha_{k i}$ is the angle between vector $\overrightarrow{L_{k i}}$ and the vertical direction.

\subsection{NETWORK MODEL}

To investigate the movement of a ganglion in a porous medium, this study developed a two-dimensional network model based on the work of Payatakes et al. (1973, 1980), and $\mathrm{Ng}$ and Payatakes (1980). In the network model, idealized pore geometry is used to contain the irregular ganglion (Figure 5a). A unit cell as depicted in Figure 5b simulates a pore tube. The wall profile of a unit cell is assumed to be a sinusoidal function in $z$. In Figure $5 \mathrm{~b}, d$ is the pore throat, $h^{\prime}$ is the pore length, and $a^{\prime}$ is the pore diameter, while $h$ is the length of one period of sinusoidal curve. Payatakes et al. (1980) gave the expressions for pore length $h^{\prime}$ and pore diameter $a^{\prime}$,

$$
\begin{aligned}
& h^{\prime}=c_{2} c_{3} d, \\
& a^{\prime}=0.5\left[\left(c_{1}+1\right)-\left(c_{1}-1\right) \cos \left(\pi c_{3}\right)\right] d,
\end{aligned}
$$

where $c_{1}, c_{2}$, and $c_{3}$ are constants that depend on grain size distribution and pore size distribution and are expressed as

$$
\begin{aligned}
& c_{1}=\left[\frac{n\left(1-S_{\mathrm{r}}\right)\left\langle d_{\mathrm{g}}^{3}\right\rangle}{1-n\left\langle d_{\mathrm{t}}^{3}\right\rangle}\right]^{1 / 3} \\
& c_{2}=\frac{\left\langle d_{\mathrm{g}}\right\rangle}{\left\langle d_{\mathrm{t}}\right\rangle}
\end{aligned}
$$

where $n$ is the porosity; $S_{\mathrm{r}}=$ residual saturation, $\left\langle d_{\mathrm{g}}^{3}\right\rangle$ the weighted average of cube of grain sizes, $\left\langle d_{t}^{3}\right\rangle$ the weighted average of cube of pore throat diameters, $\left\langle d_{\mathrm{g}}\right\rangle$ the weighted average of grain sizes, $\left\langle d_{\mathrm{t}}\right\rangle$ is the weighted average of pore throat diameters. $c_{3}$ can be calculated using 


$$
\begin{aligned}
& c_{2}\left(c_{1}+1\right)^{2} \pi c_{3}-2 c_{2}\left(c_{1}^{2}-1\right) \sin \pi c_{3}+ \\
& \quad+0.5 c_{2}\left(c_{1}-1\right)^{2}\left(\pi c_{3}+\sin \pi c_{3} \cos \pi c_{3}\right)=\frac{4 \pi c_{1}^{3}}{3},
\end{aligned}
$$

when $c_{1}$ and $c_{2}$ are known.

The two-dimensional network model is composed of randomly sized unit cells, as shown in Figure 5c. The length of periodicity of the network is

$$
l=\left[\frac{\pi}{6(1-n)}\left\langle d_{\mathrm{g}}^{3}\right\rangle\right]^{1 / 3},
$$

when the grains are spherical (Payatakes et al., 1973).

When a ganglion occupies a single cell, the unit occupation is referred as one conceptual elemental void space (CEVS). A CEVS consists of a conceptual node with no volume or resistance to flow and four halfunit-cells, as shown in Figure 5c. The volume of one EEVS in a twodimensional network model is given by unit cell

$$
V_{\mathrm{uc}}=\frac{\pi c_{1}^{3}}{12} d^{3} .
$$

The initial ganglion topology can have one or more than one CEVS, and the ganglion, if mobilized, is assumed to advance one CEVS at a step.

The pore throat diameters of the unit cells in the network are randomly assigned using the pore throat distribution (PTD) of the porous medium. After a ganglion of a certain volume is injected in the network, the ganglion topology is randomly generated, and the gate throats - the throats that are at the interface between the ganglion and the surrounding immiscible fluid - are specified. Then the appendix mobility factor $\beta_{k i}$ is calculated for each pair of upstream gate throat (indexed by $k$ ) and downstream gate throat (indexed by $i$ ), so that the $\max \beta_{K I}$ can be derived, where $K$ and $I$ are the upstream and downstream gate indices, respectively. The ganglion, if mobilized, will advance downstream through gate throat $I$, while the CEVS with gate throat $K$ at upstream will be invaded by the upstream surrounding fluid. Given the flow velocity, intrinsic permeability, density difference, interfacial tension between water and NAPL, and absolute viscosity of the wetting fluid, the ganglion fate can be determined using the mobility criterion developed in this study.

Ganglion breakup can also be studied using this network model. A breakup occurs when a ganglion fissions at a certain pore throat. To determine breakup, intermediate $\beta$ of each pair of throats (gate throat with nongate throat, nongate throat with nongate throat) is calculated. If any intermediate $\beta$ satisfies the mobilization criterion, the ganglion is considered broken up at one of the intermediate pore throats that this $\beta$ represents. It is worth mentioning that breakup at a pore throat may not 
necessarily indicate that the ganglion breaks up into two separate ganglia. When intermediate pore throat fissions, the ganglion could be broken up into two solitary ganglia or could still remain as one entity. In addition, when pore throat breakup occurs, the whole ganglion can be either mobilized or stranded.

\subsection{MODEL APPLICATION}

A two-dimensional network model with $100 \times 100$ nodes was built to simulate the pores of a porous medium. A hypothetical lognormal PTD was used in the simulation (Figure 6). Our microscopic image analysis of the glass beads samples showed that a pore size is about five times pore throat size; the study of Payatakes et al. (1973) indicated the same magnitude in the pore size and pore throat size relationship. As shown in the experimental study (Figures 3 and 4), the average pore size is about $1.0 \mathrm{~mm}$ for the $0.8 \mathrm{~mm}$ and $1.5 \mathrm{~mm}$ glass beads sample. Assuming pore size is five times pore throat size, we have the mean pore throat size of $0.2 \mathrm{~mm}$ for the $0.8 \mathrm{~mm}$ and $1.5 \mathrm{~mm}$ glass beads sample. The input parameters of the model are listed in Table II. The procedure for calculating ganglion fate is as follows:

(1) Initialize the pore throats of the network using the given PTD.

(2) Inject a ganglion of fixed volume into the network and randomly generate the ganglion topology according to the CEVS sizes and the

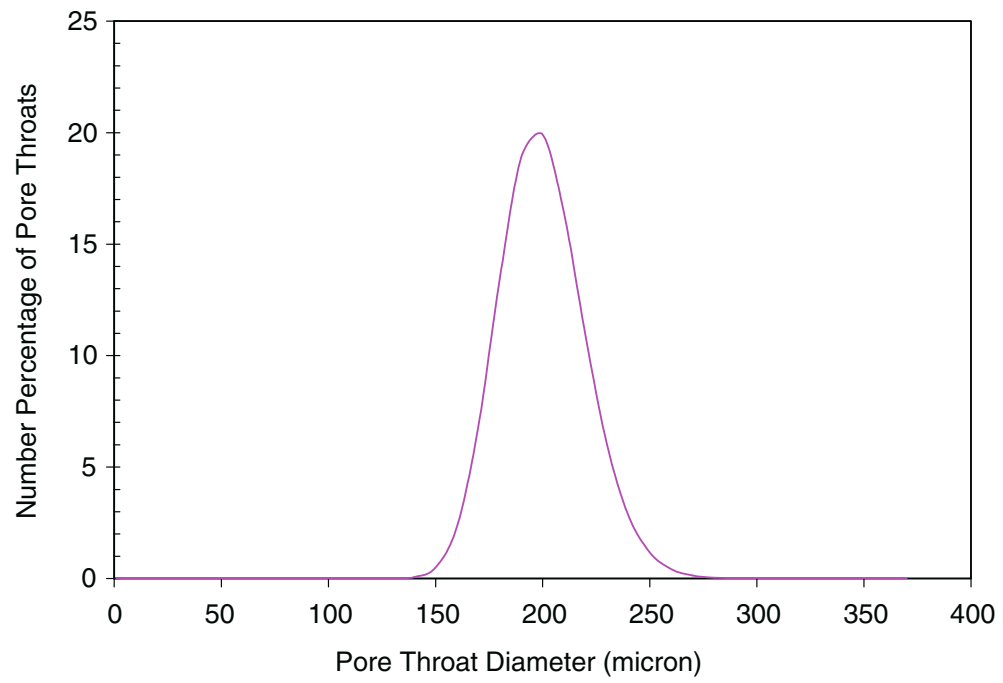

Figure 6. Hypothetical lognormal pore throat distribution $($ mean $=200 \mu \mathrm{m}$, standard deviation $=20 \mu \mathrm{m})$. 
Table II. Input parameters of the 2D network model

\begin{tabular}{ll}
\hline Absolute viscosity of water $(\eta)$ & $1.02 \times 10^{-7} \mathrm{~N} \mathrm{~s} / \mathrm{cm}^{2}$ \\
Interfacial tension between LNAPL and water $(\gamma)$ & $4.78 \times 10^{-4} \mathrm{~N} / \mathrm{cm}$ \\
Flow velocity $(v)$ & $0.001 \mathrm{~cm} / \mathrm{s}$ \\
Initial ganglion volume & $0.8 \times 10^{-4} \mathrm{ml}$ \\
Glass beads $(1.2$ and $2.0 \mathrm{~mm}$ mixture of equal & $0.1 \mathrm{~cm} / \mathrm{s}$ \\
mass) permeability $(K)$ before vibration & \\
Glass beads sample porosity $(n)$ & 0.411 \\
LNAPL density & $0.76 \mathrm{~g} / \mathrm{cm}^{3}$ \\
Length of periodicity of network $(l)$ & $2.33 \mathrm{~mm}$ \\
$c_{1}$ & 4.223 \\
$c_{2}$ & 4.460 \\
$c_{3}$ Network periodicity $(l)$ & 0.855 \\
\hline
\end{tabular}

ganglion volume. The ganglion takes about 3-15 CEVS according to the pore sizes. The initial ganglion position is the center of the network.

(3) According to the ganglion topology, specify the upstream and downstream gate throats.

(4) Using Equations (17) and (18), calculate the maximum appendix mobility factor $\left(\beta_{\max }\right)$ and specify the upstream index $K$ and the downstream index $I$ that make $\beta$ maximum.

(5) Using ganglion mobility criterion (19) and (20), determine the fate of the ganglion.

(6) Determine whether a pore throat will break up by calculating each intermediate $\beta$ and applying it to the mobility criterion.

One thousand simulation runs were carried out and each run followed the proceeding procedure, so that the mobility probabilities (mobilization, stranding, and breakup) of a ganglion could be derived. In order to study the effect of pore changes due to vibrations on ganglion movement, each pore throat in the network was perturbed in a certain range to simulate the vibration effect. The perturbation range is determined from the vibration experiments. Since the vibration amplitude is $0.33 \mathrm{~mm}$, and from Figure 3, if we take $D_{50}=1.1 \mathrm{~mm}$ as the mean pore size before vibration, we can assume that a pore size varies in a range of $1.1 \pm 0.33 \mathrm{~mm}$, or $[-0.3,0.3]$ in percentage, under vibrations. Likewise, from Figure $4, D_{50}=1.8 \mathrm{~mm}$ before vibration, we assume that a pore size varies in a range of $1.8 \pm 0.33 \mathrm{~mm}$, or $[-0.18,0.18]$ in percentage, under vibrations. In this simulation, after the original PTD was assigned to the network, each throat was perturbed in a range of $[-0.25,0.25],[-0.5,0.5]$, or $[-0.75,0.75]$ of the original pore 


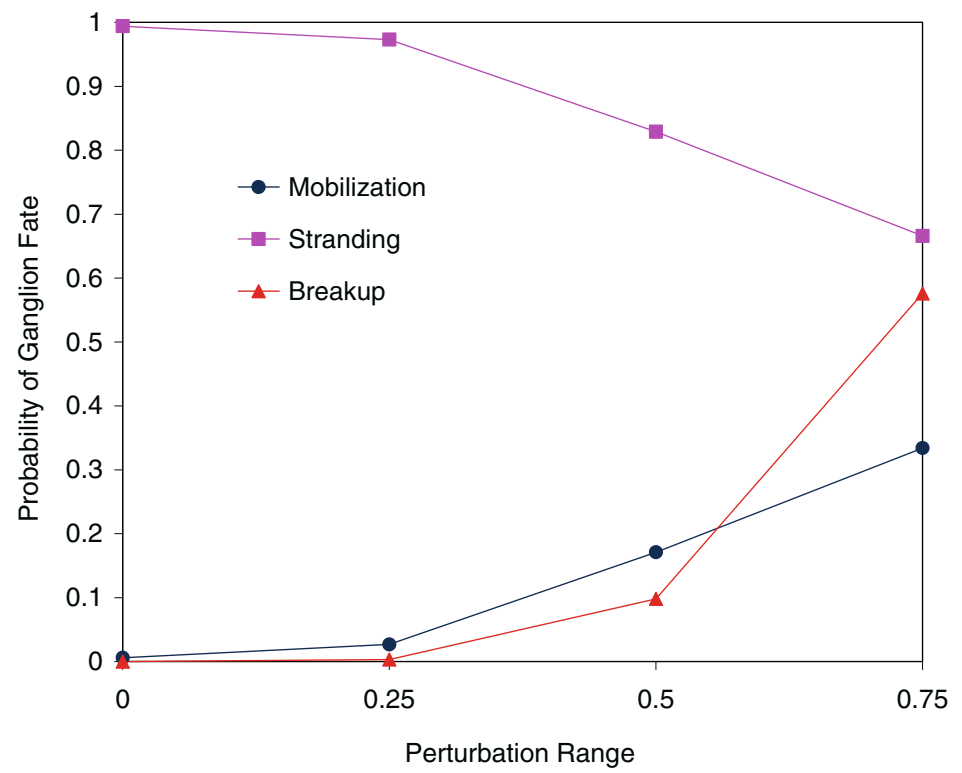

Figure 7. The effect of perturbations of pore throats on ganglion fate (pore compression probability $=40 \%$, dilation probability $=60 \%$ ).

throat to simulate different vibration intensities, and the ganglion fate is calculated using the perturbed PTD.

Figure 7 shows the modeling results in terms of probabilities of mobilization, stranding, and breakup against different perturbation ranges, using the PTD shown in Figure 6. In this simulation, each pore throat has $40 \%$ chance to shrink and $60 \%$ chance to dilate. The results indicate that the mobilization and breakup probabilities increase as perturbation range increases. Figure 8 shows the modeling results when each pore throat has $60 \%$ chance to shrink and $40 \%$ chance to dilate. Comparison of the results in Figures 7 and 8 reveals that when pore throats are more likely to shrink, viz. when a soil is compacted, a ganglion in the soil is more likely to be broken up.

To further study the role of pore throat distributions of porous media, three hypothetical PTDs shown in Figure 9 were used in the model simulation. PTD 1 has only two pore throat sizes $(0.05$ and $0.1 \mathrm{~mm})$, and each occupies $50 \%$ of the total number of pore throats; PTD 2 contains 0.15 and $0.2 \mathrm{~mm}$ pore throats, each occupies $50 \%$ of the total number of pore throats; PTD 3 consists of four different pore throat sizes $(0.05,0.1$, 0.15 , and $0.2 \mathrm{~mm}$ ), each has $25 \%$ of pore throat occupation. No perturbation is induced in this part of the study. The modeling results are shown in Figure 10, which shows similar probabilities of mobilization, stranding, and breakup for PTD 1 and PTD 2. Although PTD 1 and PTD 2 cover 


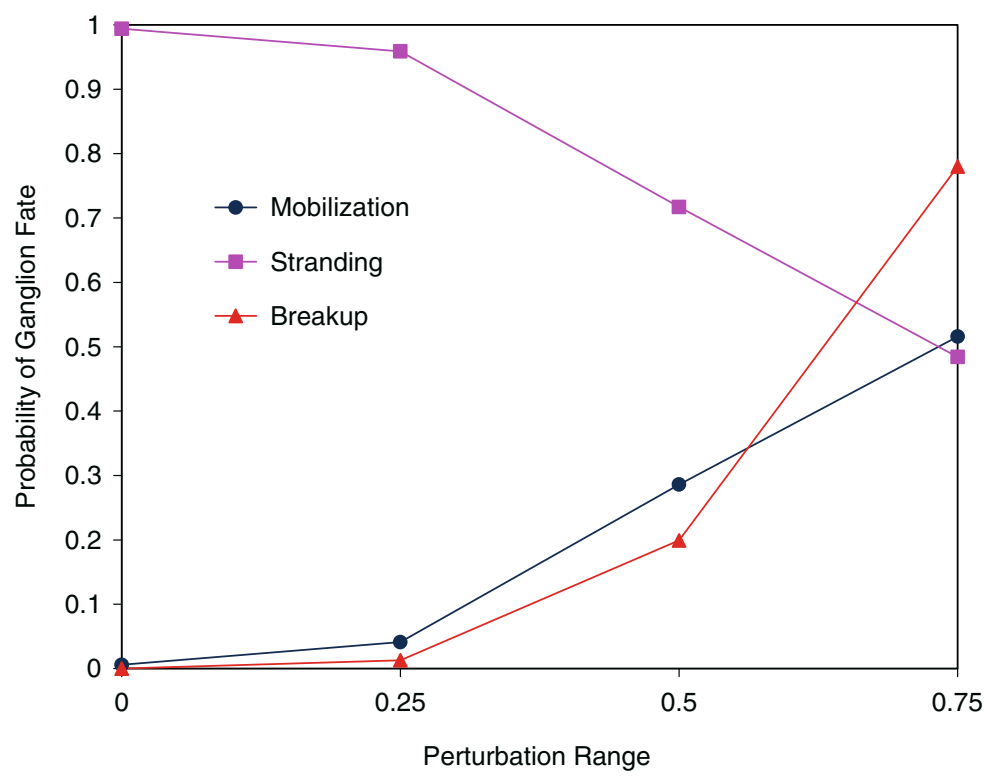

Figure 8. The effect of perturbations of pore throats on ganglion fate (pore compression probability $=60 \%$, dilation probability $=40 \%$ ).

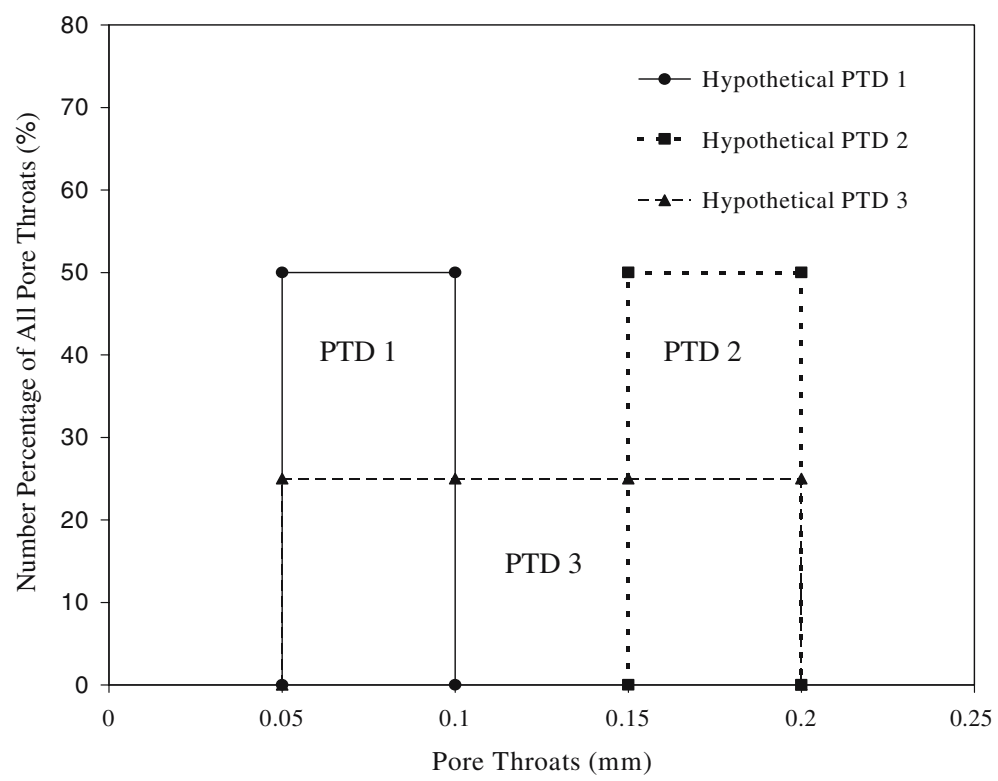

Figure 9. Hypothetical pore throat distributions (PTD) (PTD 1 covers $0.05-0.1 \mathrm{~mm}$, with two pore throat sizes, 0.05 and $0.1 \mathrm{~mm}$; PTD 2 covers $0.15-0.2 \mathrm{~mm}$, with two pore throat sizes, 0.15 and $0.2 \mathrm{~mm}$; PTD 3 covers $0.05-0.2 \mathrm{~mm}$, with four pore throat sizes, $0.05,0.10,0.15$ and $0.2 \mathrm{~mm}$ ). 


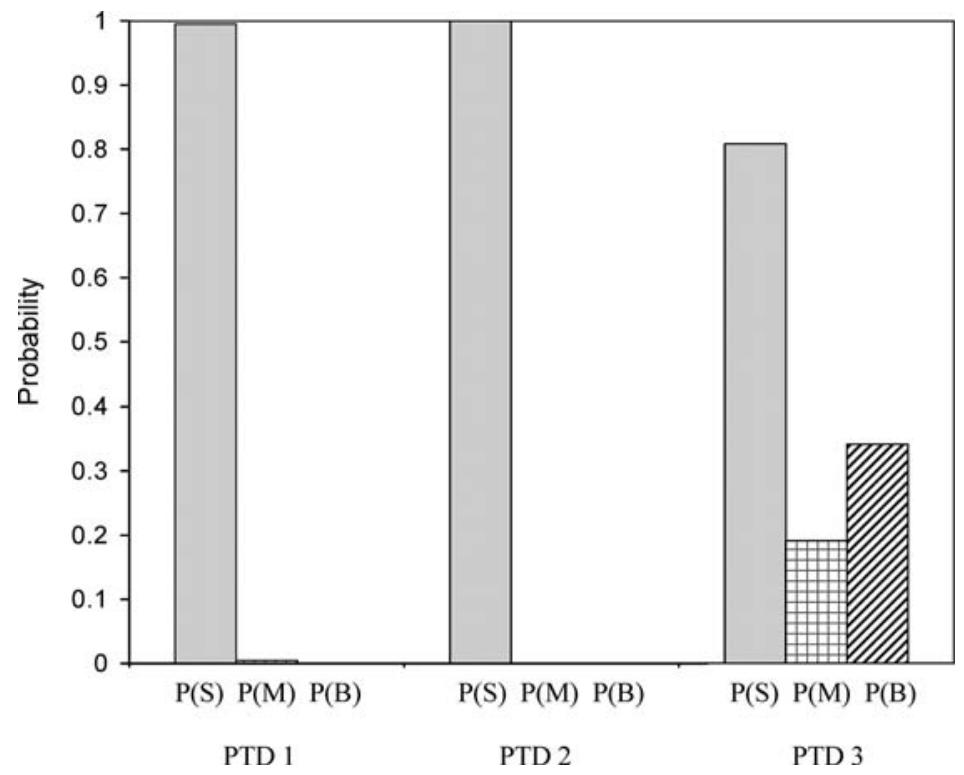

Figure 10. The effect of different pore throat distributions (PTD) on ganglion fate $(\mathrm{P}(\mathrm{S})$ - Stranding probability; $\mathrm{P}(\mathrm{M})$ - mobilization probability; $\mathrm{P}(\mathrm{B})$ - breakup probability).

different pore throat ranges, the pore throat differences are the same. As suggested by Equation (15), ganglion fate is determined by the difference of pore throat curvature. Therefore, the ganglion fates (mobilization, stranding, or breakup) from both PTD 1 and PTD 2 are similar. PTD 3 has bigger pore throat size difference, and it yielded noticeably different ganglion fate. The modeling results suggest that ganglion fate is not controlled by the pore throat sizes, but by the range of variation in pore throat sizes. A ganglion in a porous medium with more uniform pore throat distribution is less likely to be mobilized and broken up.

It should be noted that the current model only gives the initial mobilization, breakup, and stranding probabilities of a solitary ganglion. $\mathrm{Ng}$ et al. (1978) and Reddi et al. (1998) observed subsequent ganglia breakup, collision, and coalescence in laboratory. Ganglia population studies are necessary to account for subsequent ganglia breakup and coalescence.

\section{Conclusions}

In summary, both experimental and modeling results point to the general conclusion that vibrations of porous media tend to break up discontinuous ganglia. The following are specific conclusions drawn from this study: 
(1) Distribution of wetting fluid at residual saturation in the form of discontinuous blobs can be represented using van Genuchten expression.

(2) The effect of vibrations is to break up blobs and reduce the mean blob size. The size distributions of discontinuous pore fluid after vibrations are less uniform than those before vibrations.

(3) Modeling shows that as vibration intensifies, a solitary pore fluid is more likely to be broken up.

(4) The modeling investigation also reveals that ganglion fate in a porous medium may be controlled by the range of variation in pore throat sizes as opposed to absolute sizes of pore throats.

\section{Acknowledgements}

This research is funded by the Advanced Life Support Program of NASA John Space Center (Project Number: NAG9-1399). The support from this agency is gratefully acknowledged. The authors thank the anonymous reviewers for their constructive comments that help to improve the paper.

\section{References}

Mayer, A. S. and Miller, C. T.: 1992, The influence of porous medium characteristics and measurement scale on pore-scale distributions of residual nonaqueous-phase liquids, J. Contaminant Hydrol. 11, 189-213.

Ng, K. M., Davis, H. T. and Scriven, L. E.: 1978, Visualization of blob mechanics in flow through porous media, Chem. Engng. Sci. 33, 1009-1017.

Ng, K. M. and Payatakes, A. C.: 1980, Stochastic simulation of the motion, breakup and stranding of oil ganglia in water-wet granular porous media during immiscible displacement, AIChe J. 26(3), 419-429.

Payatakes, A. C., Ng, K. M. and Flumerfelt, R. W.: 1980, Oil ganglion dynamics during immiscible displacement: Model formulation, AIChe J. 26(3), 430-442.

Payatakes, A. C., Tien, C. and Turian, R. M.: 1973, A new model for granular porous media: Part I. model formulation, AIChe J. 19(1), 58-67.

Reddi, L. N. and Challa, S.: 1994, Vibratory mobilization of immiscible liquid ganglia in sands, J. Environ. Engng. 120(5) 1170-1190.

Reddi, L. N, Menon, S. and Pant, A.: 1998, Pore-scale investigation on vibratory mobilization of LNAPL ganglia, J. Hazard. Materials 62, 211-230. 